Check for updates

Cite this: RSC Adv., 2017, 7, 19085

Received 1st January 2017

Accepted 13th March 2017

DOI: $10.1039 / \mathrm{c} 7 \mathrm{ra00010c}$

rsc.li/rsc-advances

\section{Study of dielectric relaxation and thermally activated a.c. conduction in lead containing topological glassy semiconductors $\uparrow$}

\begin{abstract}
Arvind Sharma ${ }^{a}$ and N. Mehta (iD *b
Multi-component topological glasses of the $\mathrm{Te}_{20} \mathrm{Sn}_{2} \mathrm{Se}_{78-x} \mathrm{~Pb}_{x}(0 \leq x \leq 6)$ system have been prepared by a cost-effective melt-quench technique that is well-known for its convenience. The study of dielectric relaxation in this novel glassy system was investigated as a function of temperature in the 300-350 K temperature regime and $50 \mathrm{kHz}$ to $1 \mathrm{MHz}$ frequency regime. An extensive investigation proved that dielectric losses are dipolar in nature and can be implicit in terms of hopping of charge carriers over a potential barrier, as suggested by Elliot for the case of chalcogenide materials. It was found that both dielectric constant and dielectric loss depend on temperature and frequency. Moreover, it was found that the values of both dielectric constant $\varepsilon^{\prime}$ and dielectric loss $\varepsilon^{\prime \prime}$ decrease with frequency and increase with increasing temperature. A detailed analysis showed that dielectric loss $\varepsilon^{\prime \prime}$ obeys Guintini relation. The variation in the dielectric properties and a.c. conductivity with $\mathrm{Pb}$ composition was also explored. Density of defect states in the present glasses was measured using a correlated barrier hopping $(\mathrm{CBH})$ model. According to the $\mathrm{CBH}$ model, electron hopping takes place over the coulombic barrier height $W$ and neighbouring sites at a distance, $R$. The Coulomb wells overlap and result in a lowering of the effective barrier height from $W_{\mathrm{m}}$ to a value, $W$.
\end{abstract}

\section{Introduction}

Chalcogenide glassy semiconductors (ChGS) have come under scientific scrutiny in the recent two decades due to their potential applications in optoelectronics and optics. ${ }^{\mathbf{1 - 8}}$ These materials exhibit flexible structure, enormous variation in properties, and almost unlimited ability for doping and alloying for chemical sensors in the investigation of industrial aqueous solutions to monitor pollutant gasses. ${ }^{\mathbf{1}}$ Furthermore, ChGS are the recognized candidates for utilization in fiber optics and integrated optics due to their excellent transparency in the infrared region, ${ }^{2}$ electronic and optical switching ${ }^{3,4}$ and optical recording media. ${ }^{5-7}$ Due to their IR transparency, photosensitivity, and high optical nonlinearity, these materials have been utilized to fabricate photonic devices such as fibers, ${ }^{7}$ planar waveguides, and ${ }^{8}$ gratings. The fundamental assets of ChGS can be modified as per the necessity of the technological applications. Incorporating metallic substituents alters the necessary bonding network and bonding structures of the material.

Incorporating $\mathrm{Pb}$ into the glassy network is a way to tune its physico-chemical properties by improving thermal stability and

${ }^{a}$ School of Physical and Material Sciences, Mahatma Gandhi Central University, Motihari-845401, India

${ }^{b}$ Department of Physics, Institute of Science, Banaras Hindu University, Varanasi221005, India.E-mail: dr_neeraj_mehta@yahoo.co.in

$\dagger$ PACS.72.80.Ng disordered solids - 61.43.Fs glasses. glass forming ability by lowering the aging effects., ${ }^{9,10}$ Addition of lead at a specific composition in some ChGS shows carriertype reversal. ${ }^{11}$ In recent years, $\mathrm{Pb}$ - and Sn-containing glasses $^{9-11}$ and topological crystalline insulators ${ }^{12-14}$ of Se and Se-Te system have drawn attention of scientific community due to their interesting, significant and novel properties.

Dielectric and a.c. conductivity measurements in various types of advanced functional materials are of particular importance, not only from an application point of view but also from a fundamental point of view. ${ }^{15-17}$ In addition, a study of temperature dependence of dielectric permittivity, particularly in the range of frequencies where dielectric dispersion occurs, can be of great importance for understanding the nature and origin of losses occurring in these materials. Moreover, the assimilation of some metallic elements to the chalcogenide glasses causes enriched conductivity, followed by producing a substantial decrease in activation energy for transporting electrical conduction. ${ }^{18}$ This feature makes them more suitable for integrated circuits (ICs) device applications. A sensibly comprehensive understanding of electrical transport properties of ChGS is, therefore, a prime goal and demand of glass science and technology to provide relevant information for their practical utilization in the field of solid state devices. With this research plan in mind, we prepared a novel multi-component ChGS of Se-Te-Sn-Pb system.

In fact, our previous studies on ChGS of Se-Te-Sn showed that adding Sn to the Se-Te system is an efficient tool to 
enhance its dielectric dispersion. ${ }^{19}$ The reason behind the selection of $\mathrm{Pb}$ as a modifier is the unique optical, electrical and thermal properties of lead-containing chalcogenide glasses due to the small band gap and large Bohr radius of $\mathrm{Pb}$. Due to this feature, lead chalcogenides become a better candidate for a tremendous number of applications. ${ }^{20-29}$ Examples of their potential applications are solar cells [20-221], telecommunications, ${ }^{23}$ field effect transistors (FET), ${ }^{24}$ and sensitive membrane materials for developing conventional chemical sensors selective to metal ions in liquid media, as well as biological imaging. ${ }^{25,26}$

The aim of the present investigation was to study the effect of Se atom replacement by $\mathrm{Pb}$ atoms on the electrical properties of a multi-component system. We observed that when $\mathrm{Pb}$ was incorporated into ternary parent $\mathrm{Se}_{78} \mathrm{Te}_{20} \mathrm{Sn}_{2}$ glass, then the thermal stability and glass forming ability of quaternary samples was enhanced noticeably. This communication deals with frequency and temperature dependence of the dielectric properties of multi-component ChGS of the $\mathrm{Te}_{20} \mathrm{Sn}_{2} \mathrm{Se}_{78-x} \mathrm{~Pb}_{x}(0$ $\leq x \leq 6)$ system over the 303-348 K temperature range and 50 $\mathrm{kHz}$ to $1 \mathrm{MHz}$ frequency range. We have seen the influence of impurity element $\mathrm{Pb}$ into the host glassy $\mathrm{Se}_{78} \mathrm{Te}_{20} \mathrm{Sn}_{2}$ alloy on the dielectric properties and a.c. conductivity.

\section{Material synthesis and characterization}

The multi-component ChGS of the present system was synthesized by the usual melt quenching technique, and the details of this quaternary system have been described elsewhere. ${ }^{2,19}$ The glassy nature of the aforesaid system has been confirmed by the X-ray diffraction technique (XRD). For this, a standard X-ray diffractometer with $\mathrm{CuK}_{\alpha}$ radiation $(\lambda=$ $1.5405 \AA$ A) was employed. The typical XRD patterns of all the samples are shown in Fig. 1. The existence of crystalline-like ordering in the melt-quenched glasses is still a subject of debate in the literature. ${ }^{30}$ Therefore the presence of some blunt peaks in the XRD patterns creates a doubt regarding the possibility of crystallized fractions, but this can be regarded as negligible since the corresponding peak intensities are very small or; in other words, these XRD patterns show diffraction peaks without any signatures of crystalline phases on the macro-scale.

Surface morphology characterization of the synthesized material was carried out by employing a scanning electron microscope (SEM) (Model: Quanta 200F) with an operating voltage of $25 \mathrm{keV}$ (see Fig. 2).

Fig. 3 shows characteristic DSC scans of the topological parent ternary and $\mathrm{Pb}$-containing quaternary glasses. This figure reveals that the present samples underwent distinctive structural relaxation and crystallization phenomena through well defined endothermic and exothermic peaks in the region of the peak glass transition temperature $T_{\mathrm{g}}$ and peak crystallization temperatures, respectively. The appearance of these peaks was the further confirmation of the overall glassy nature of the present topological semiconductors.

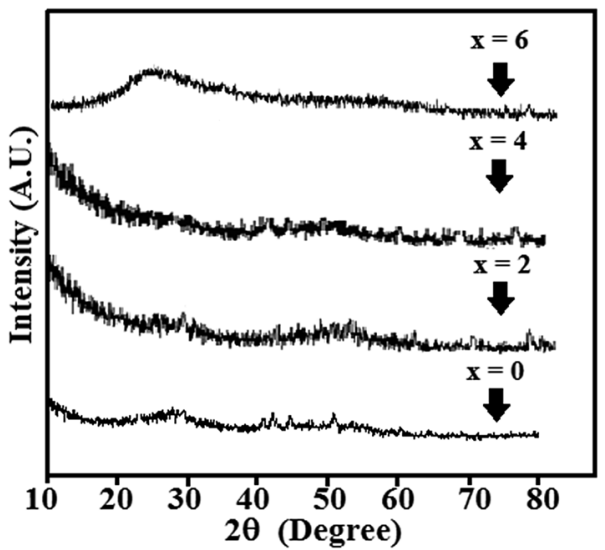

Fig. 1 XRD pattern of the glassy $\mathrm{Te}_{20} \mathrm{Sn}_{2} \mathrm{Se}_{78-x} \mathrm{~Pb}_{x}(0 \leq x \leq 6)$ system.

For dielectric measurements, the aforementioned prepared glassy systems were ground to a very fine powder, and pellets (diameter $\sim 10 \mathrm{~mm}$ and thickness $\sim 1 \mathrm{~mm}$ ) were obtained by compressing the powder in a die at a 5 Ton load. The pellets were mounted between two smooth aluminum electrodes of a metallic sample holder for dielectric measurements.

The sample holder employed in the present measurements consisted of two portions (see Fig. 4). The upper portion had two electrodes and an evacuation port. The samples were mounted between the jaws of two electrodes having a spring arrangement. At the end of the two electrodes, two UHF connectors were used for the electrical connections to the sample. Kanthal wire was wound as a heating element on the outer covering of the sample holder assembly (Fig. 4(b)). The heating element was covered with Plaster of Paris in order to avoid heat losses due to radiation. An O-ring was provided between the two parts of the sample holder for proper evacuation inside the chamber. The pellets were coated with silver paint to ensure good electrical contact between the sample and the electrodes.

The temperature measurement was facilitated by a copper constantan thermocouple mounted very near to the sample. A vacuum of $10^{-3}$ Torr was continued over the entire temperature range (303-348 K). Dielectric measurements were made using a digital LCR meter (Wayne Kerr Electronics, Model: 43100). The parallel capacitance and dissipation factor were measured, and $\varepsilon^{\prime}$ and $\varepsilon^{\prime \prime}$ were calculated. The authors favored to experiment on the pellet since the bulk had potential macroscopic effects (gas bubbles, etc.) that may have appeared during preparation. As was previously shown, ${ }^{28}$ both theoretically and experimentally, the bulk ingots and compressed pellets exhibit similar dielectric behavior in chalcogenide glasses for the suspected inhomogeneities in the case of compressed pellets for these materials.

\section{Theoretical basis}

Guintini and his co-workers ${ }^{31}$ suggested a suitable model for the study of dielectric dispersion in terms of the variation of the dielectric loss with the frequency and/or the temperature range as follows: 

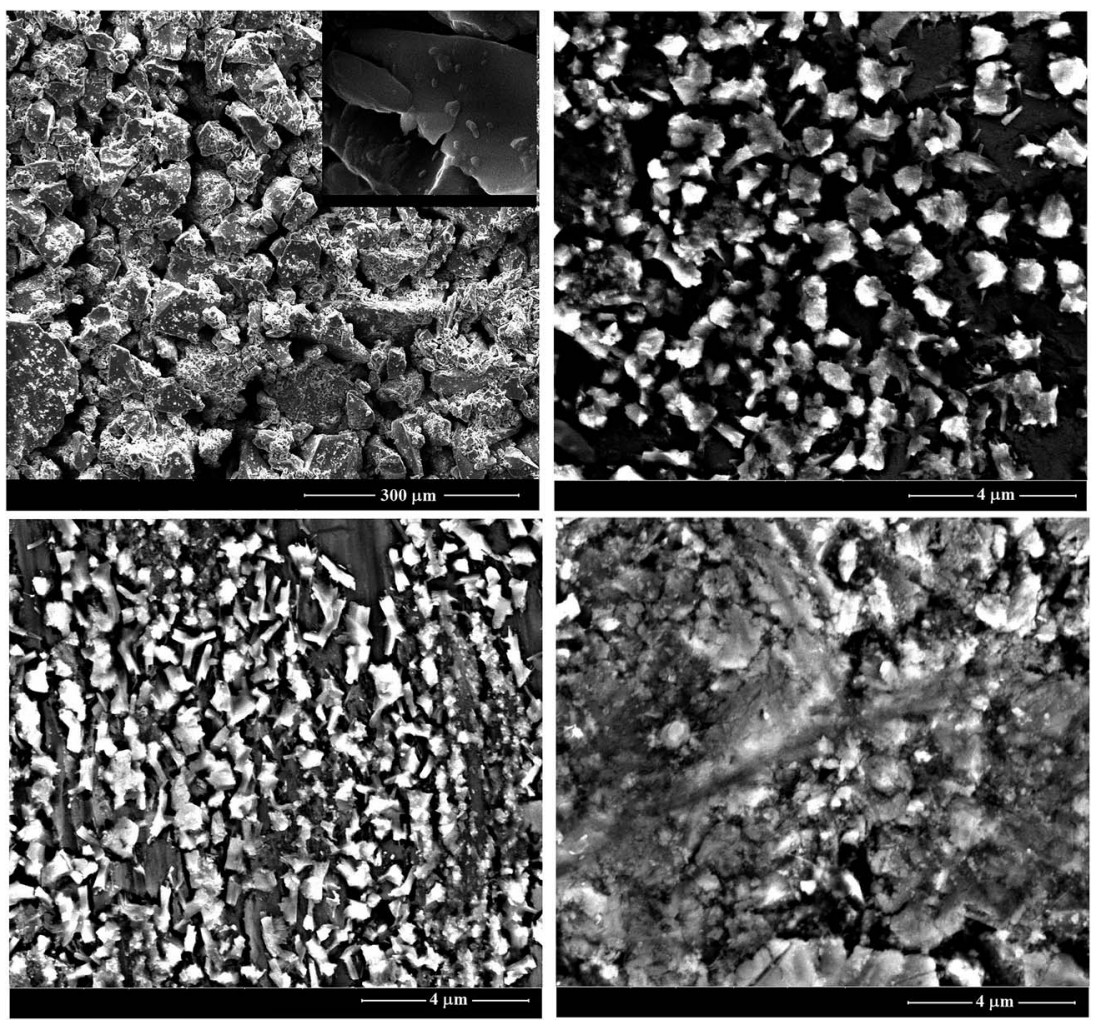

Fig. 2 SEM images for the glassy $\mathrm{Te}_{20} \mathrm{Sn}_{2} \mathrm{Se}_{78-x} \mathrm{~Pb}_{x}(0 \leq x \leq 6)$ system. Here SEM image is shown in left side of the upper panel for $x=0$, SEM image is shown in the right side of the upper panel for $x=2$, SEM image is shown in the left side of the lower panel for $x=4$ and the SEM image is shown in the right side of the lower panel for $x=6$.

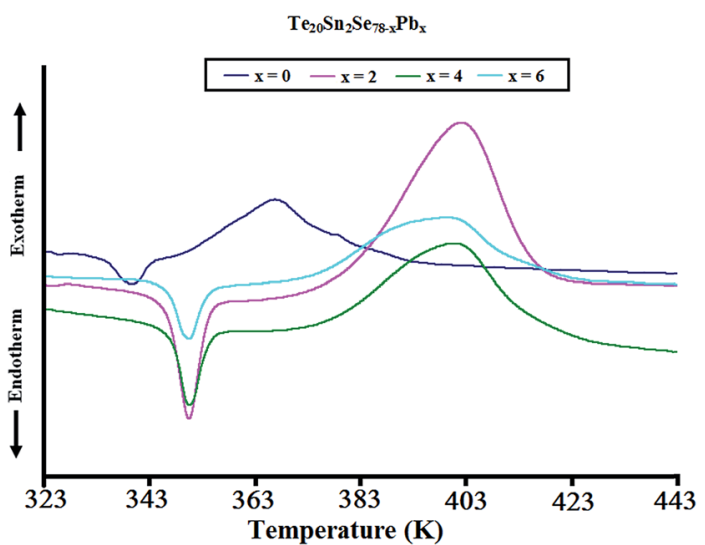

Fig. 3 Characteristics DSC scans for the glassy $\mathrm{Te}_{20} \mathrm{Sn}_{2} \mathrm{Se}_{78-x} \mathrm{~Pb}_{x}(0 \leq$ $x \leq 6)$ system.

$$
\varepsilon^{\prime \prime}(\omega)=\left(\varepsilon_{0}-\varepsilon_{\infty}\right) 2 \pi^{2} N\left(\frac{n e^{2}}{\varepsilon_{0}}\right)^{3} k T \tau_{0}{ }^{m} W_{\mathrm{m}}{ }^{-4} \omega^{m}
$$

In this model, $n$ is the number of electrons that hop, $N$ is the concentration of localized sites, $\varepsilon_{0}$ is the static dielectric constant, $\varepsilon_{\infty}$ is the dielectric constant at infinitely high frequencies, and $W_{\mathrm{m}}$ is the energy required to move the electron from one site to an infinite distance. This above equation can be formed in another way as follows:

$$
\varepsilon^{\prime \prime}=B \omega^{m}
$$

where $B$ is a constant, and the frequency exponent $m$ that has negative values is expressed as follows:

$$
m=-\frac{4 k T}{W_{\mathrm{m}}}
$$

The study of thermally activated a.c. conduction in ChGS was carried out by a correlated barrier hopping $(\mathrm{CBH})$ model. This model suggests a random distribution of the localized states and the "to-and-fro" motion of the electronic charged carriers through a rigorous relaxation time between a pair consisting of two localized states. In ChGS, the total contribution to a.c. conductivity comes from the sum of all of such pairs.

The expression for the frequency dependency of a.c. conductivity in ChGS is as follows: $:^{32}$

$$
\sigma_{\mathrm{ac}}(\omega)=A \omega^{s}
$$

where $\omega$ is the angular frequency and $s$ is the frequency exponent. The value of $s$ is less than or equal to unity. In the $\mathrm{CBH}$ model, the electrons in the charged defect states hop over the coulombic barrier height $W$ and neighbouring sites at a distance $R$. The Coulomb wells overlap and result in a lowering of the effective barrier height from $W_{\mathrm{m}}$ to a value $W$ according to the following expression: 


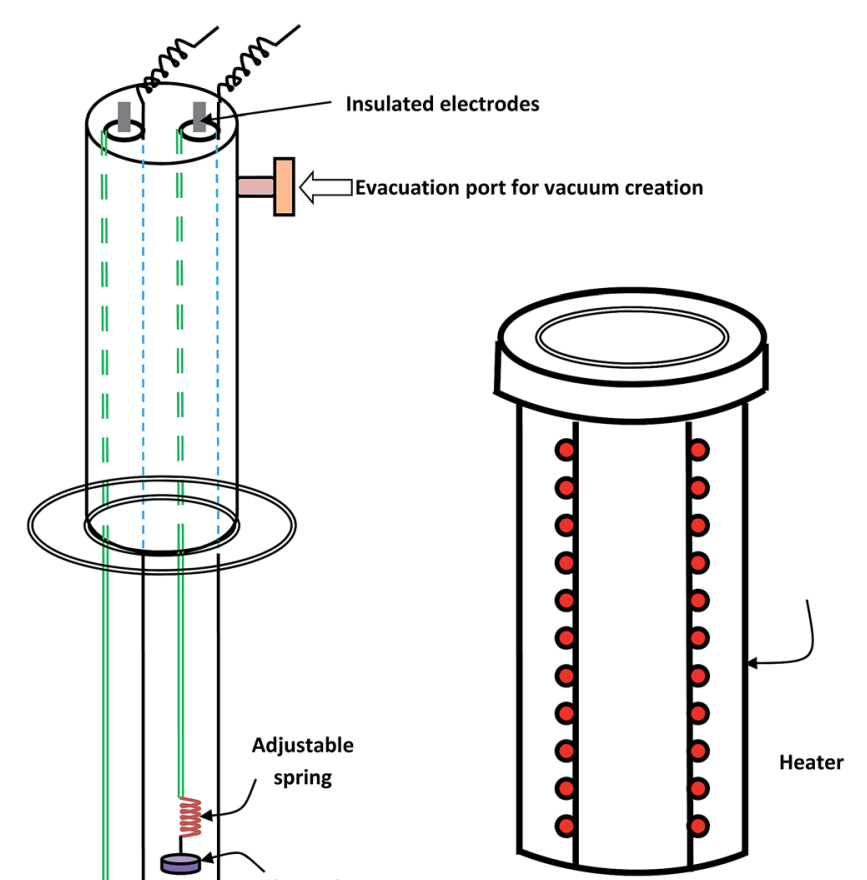

(b) Lower section of sample holder

where $\tau_{0}$ is characteristic relaxation time having the order of an atomic vibrational period, and $k$ is the Boltzmann constant $\left(1.38 \times 10^{-23} \mathrm{~J} \mathrm{~K}^{-1}\right)$. According to the $\mathrm{CBH}$ model, the a.c. conductivity $\sigma_{\mathrm{ac}}(\omega)$ for bi-polaron hopping initiating from intimate $\mathrm{D}^{+}-\mathrm{D}^{-}$pairs having a non-random distribution and can be written as follows:

$$
\sigma_{\mathrm{ac}}(\omega)=\left(\frac{n \pi^{3}}{6}\right) N N_{\mathrm{p}} \varepsilon \varepsilon_{0} \omega R_{\omega}{ }^{6} \exp \left(\frac{e^{2}}{4 \pi \varepsilon \varepsilon_{0} R_{\omega} k T_{\mathrm{g}}}\right)
$$

where $N$ is the density of localized states at which the carriers exist, $N_{\mathrm{p}}$ is the density of localized states to which the carriers hop, $T_{\mathrm{g}}$ is the glass transition temperature, and $R_{\omega}$ is the hopping distance for the condition $\omega \tau=1$ and is given by the following expression:

$$
R_{\omega}=\frac{2 e^{2}}{\pi \varepsilon \varepsilon_{0} W_{\mathrm{m}}}\left[1+\frac{k_{B} T}{W_{\mathrm{m}}} \ln \left(\omega \tau_{0}\right)\right]^{-1}
$$

The a.c. conductivity, $\sigma_{\mathrm{ac}}(\omega)$ for single polaron hopping originating from randomly distributed defect centers can be written as follows: ${ }^{32-34}$

$$
\sigma_{\mathrm{ac}}(\omega)=\frac{\pi^{3}}{6} N N_{\mathrm{p}} \varepsilon \varepsilon_{0} \omega R_{\omega}{ }^{6}
$$

The corresponding value of the hopping distance is as follows:

$$
R_{\omega}=\frac{e^{2}}{\pi \varepsilon \varepsilon_{0} W_{\mathrm{m}}}\left[1+\frac{k_{B} T}{W_{\mathrm{m}}} \ln \left(\omega \tau_{0}\right)\right]^{-1}
$$
measurements of disc shaped samples (pellets) under vacuum at $\sim 10^{-3}$ Torr.

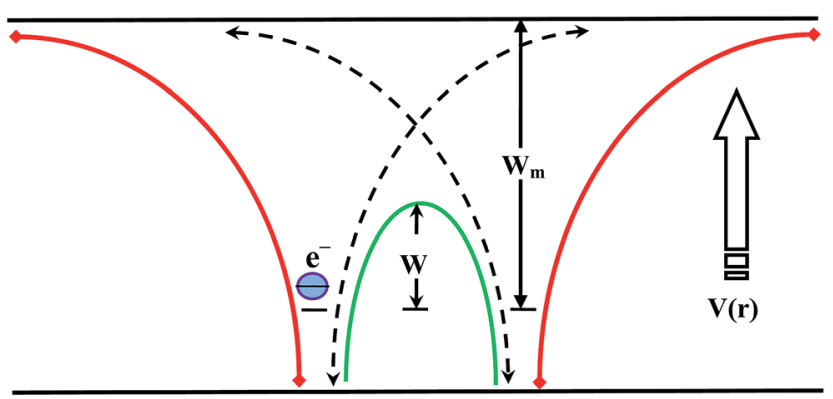

$-d R_{\omega} / 2 p$

Fig. 5 Illustration of $\mathrm{CBH}$ model of the coulombic well.

$$
W=W_{\mathrm{m}}-\frac{n e^{2}}{\pi \varepsilon \varepsilon_{0} R}
$$

This fact is shown in Fig. 5 . Here, $n$ represents the number of electrons involved in a hop $(n=1$ and $n=2$ for the singlepolaron and bi-polaron processes, respectively).

The relaxation time $\tau$ for the electrons to hop over a coulombic barrier having height $W$ is given as follows:

$$
\tau=\tau_{0} \exp \left(\frac{W}{k T}\right)
$$

If we denote the respective contributions of single-polaron hopping originating from randomly distributed defect states to a.c. conductivity and bi-polaron hopping originating from non-random distribution of defect states by $\left[\sigma_{\mathrm{ac}}(\omega)\right]_{\mathrm{s}}$ and $\left[\sigma_{\mathrm{ac}}(\omega)\right]_{\mathrm{b}}$, then the a.c. conductivity of ChGS can be determined by the sum of these two contributions, i.e.,

$$
\sigma_{\mathrm{ac}}(\omega)=\left[\sigma_{\mathrm{ac}}(\omega)\right]_{\mathrm{s}}+\left[\sigma_{\mathrm{ac}}(\omega)\right]_{\mathrm{b}}
$$

\section{Results and discussion}

\subsection{Analysis of surface morphology}

Fig. 2(a-d) shows the microstructures of the as-prepared ChGS of the $\mathrm{Te}_{20} \mathrm{Sn}_{2} \mathrm{Se}_{78-x} \mathrm{~Pb}_{x}(0 \leq x \leq 6)$ system. Image 2(a) exhibits a cluster like morphology at the same resolution of $300 \mu \mathrm{m}$, which gives a dimension of around $100 \mu \mathrm{m}$ for the pure host clusters. The morphology of the same composition at high resolution is shown in the inset of this image. Image $2(\mathrm{~b})$, taken at $4 \mu \mathrm{m}$ resolution, explores comparatively more homogeneous clusters of several tenths of $\mu \mathrm{m}$ dimensions and shows a hierarchical surface morphology. Thus, it can be concluded that an addition of 2 atomic weight percentage (at. $\mathrm{wt} \%$ ) of $\mathrm{Pb}$ content into the host material gives some un-agglomerated and hierarchical distribution of clusters of smaller size. The microstructure 2(c) shows the surface morphology of 4 at. wt $\% \mathrm{~Pb}$ content incorporated into host material at a resolution of $4 \mu \mathrm{m}$. 

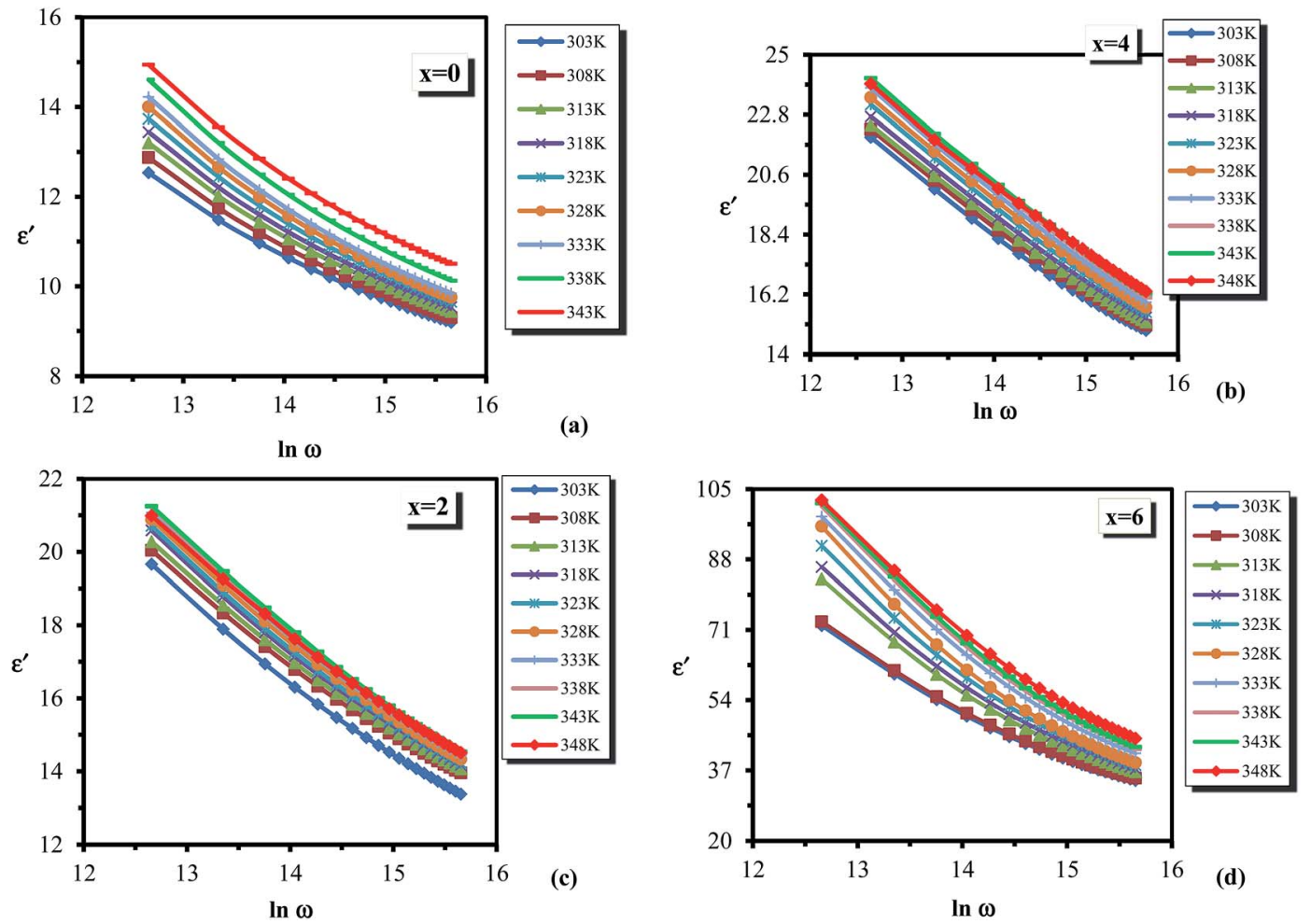

Fig. 6 Frequency dependence of dielectric constant $\varepsilon^{\prime}$ for the glassy $\mathrm{Te}_{20} \mathrm{Sn}_{2} \mathrm{Se}_{78-x} \mathrm{~Pb}_{x}(0 \leq x \leq 6)$ system.

This image shows the clusters of nearly the same dimensions as in 2(b) but they come closer to each other following hierarchy in the surface morphology. This change in morphology appears to be due to the addition of 2 at. $\mathrm{wt} \%$ of $\mathrm{Pb}$ impurity that causes a change in chemical properties of host material. Furthermore, addition of 6 at. wt $\%$ of $\mathrm{Pb}$ content into the host material breaks the $\mathrm{Pb}$ acceptance capability of the host material, which results in a drastic change in the morphology, as shown in image 2(d)
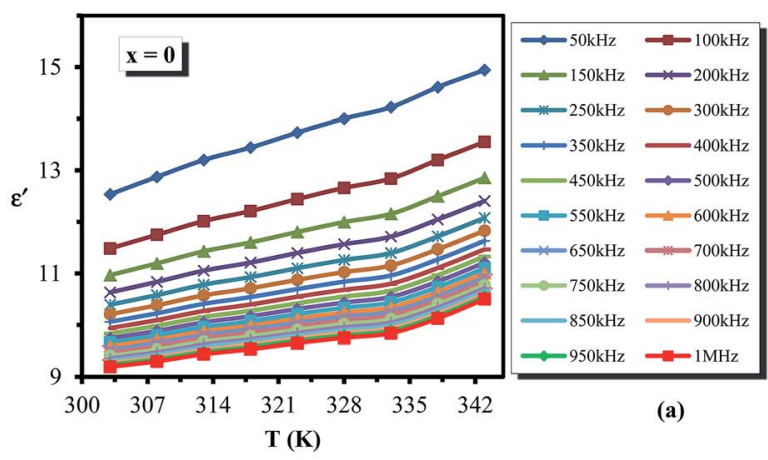

(a)
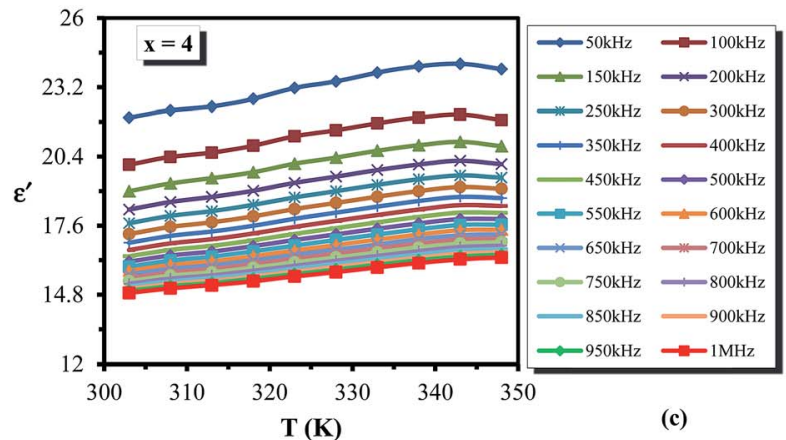
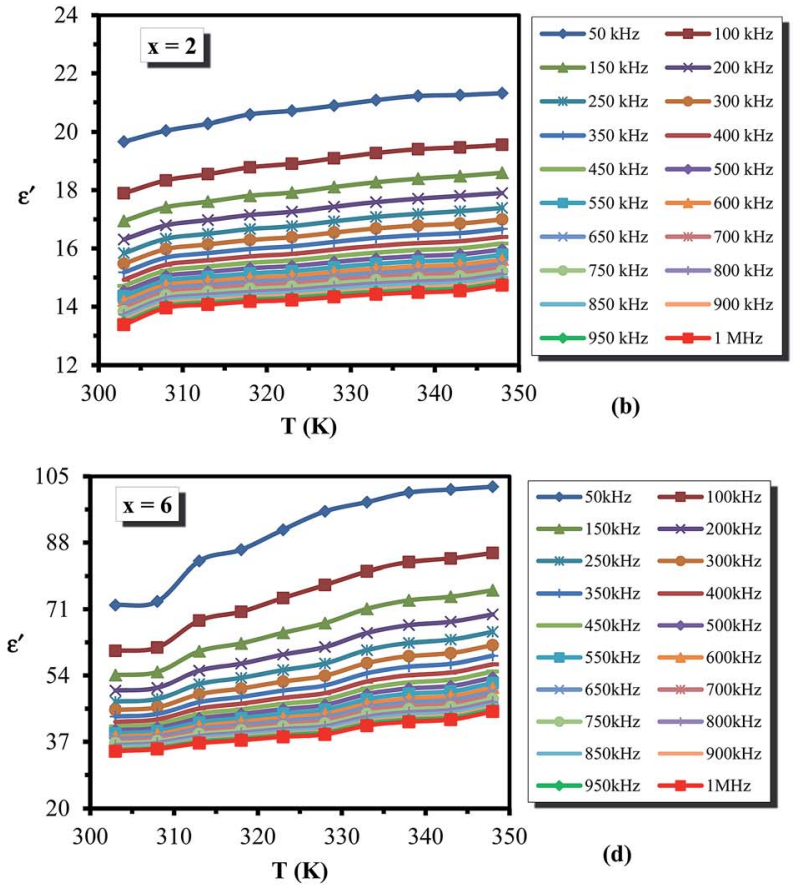

Fig. 7 Temperature dependence of dielectric constant $\varepsilon^{\prime}$ for the glassy $\mathrm{Te}_{20} \mathrm{Sn}_{2} \mathrm{Se}_{78-x} \mathrm{~Pb}_{x}(0 \leq x \leq 6)$ system. 

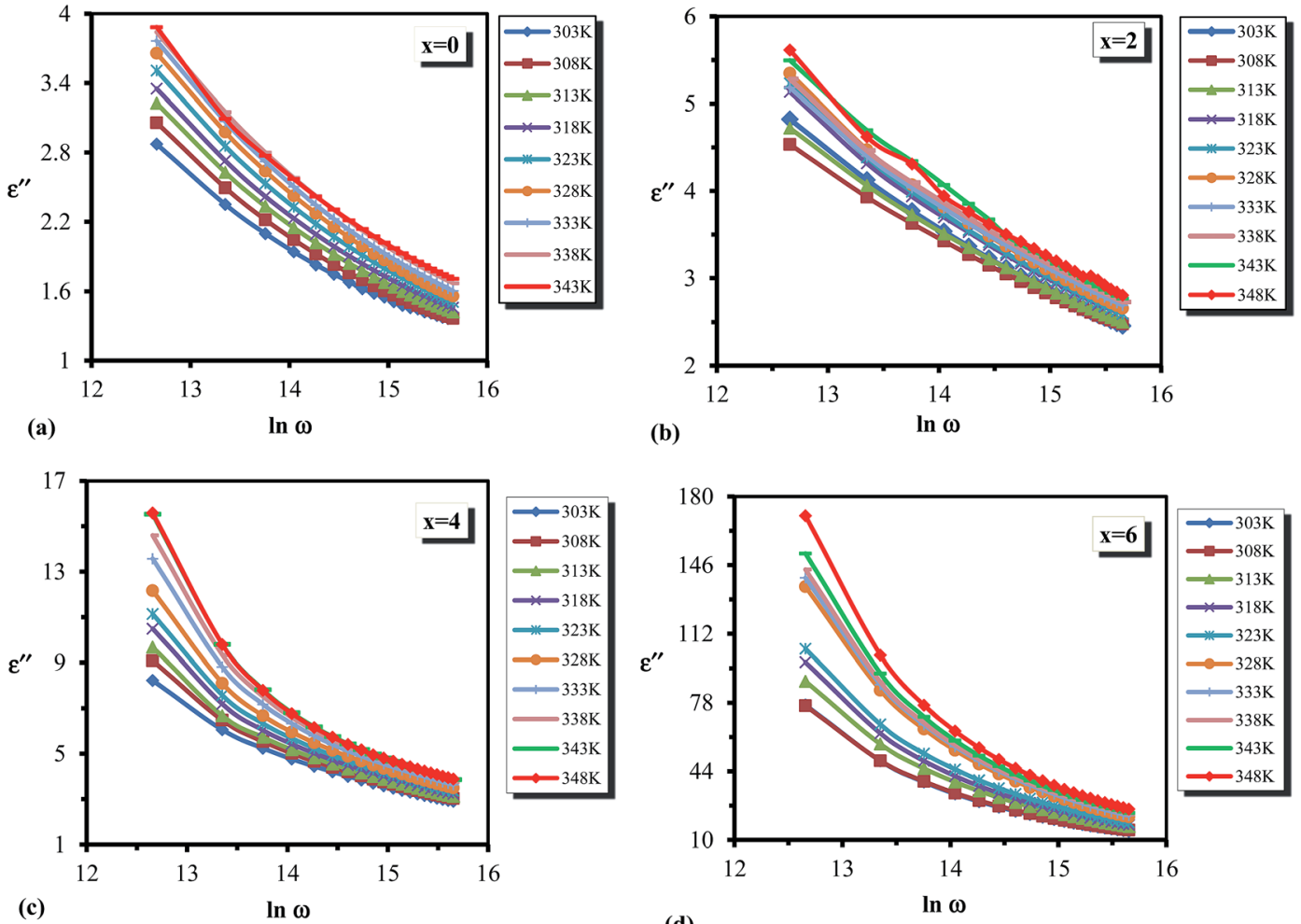

(d)

Fig. 8 (a-d) Frequency dependence of dielectric loss $\varepsilon^{\prime \prime}$ for the glassy $\mathrm{Te}_{20} \mathrm{Sn}_{2} \mathrm{Se}_{78-x} \mathrm{~Pb}_{x}(0 \leq x \leq 6)$ system.

taken at the same resolution of $4 \mu \mathrm{m}$. In this image, the clusters are almost the same size as in 2(c) and are embedded into a base material that appears to be in another phase. Upon careful examination of these images, one can see that the hierarchical style of distribution of clusters was maintained up to 4 at. wt $\%$ of $\mathrm{Pb}$ into the host material and is disrupted at 6 at. $\mathrm{wt} \%$ of $\mathrm{Pb}$.
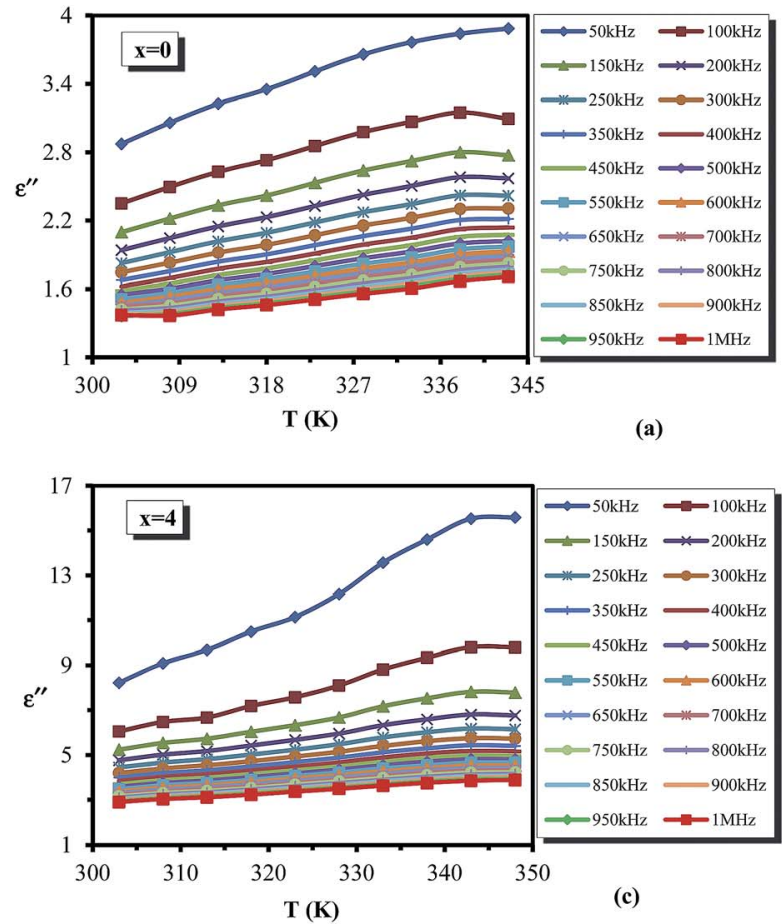
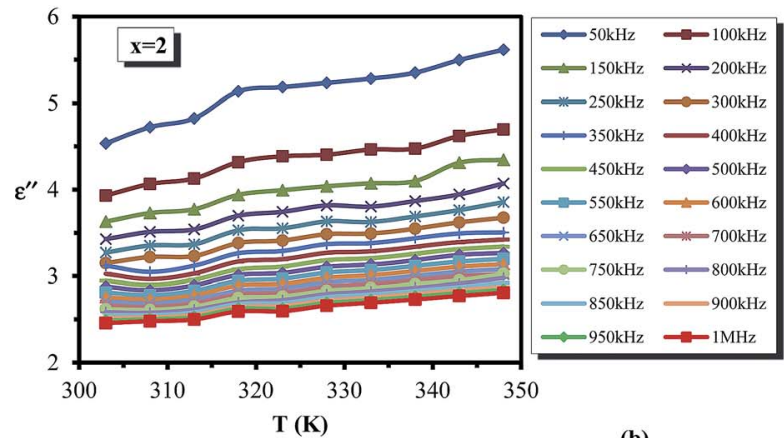

(b)
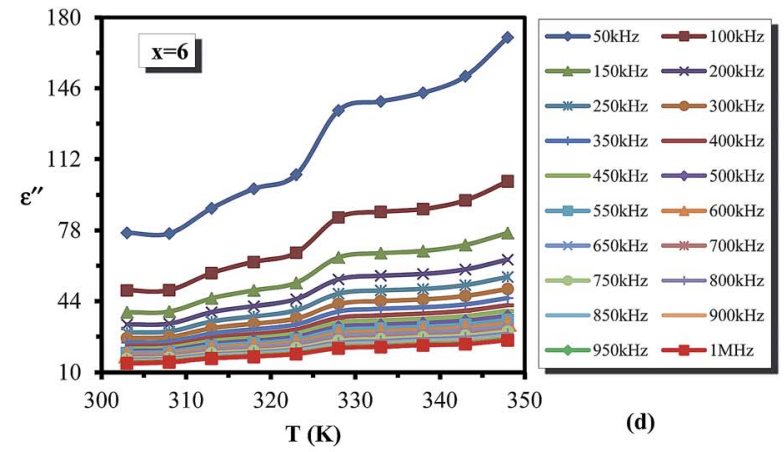

Fig. $9(\mathrm{a}-\mathrm{d})$ Temperature dependence of dielectric loss $\varepsilon^{\prime \prime}$ for the glassy $\mathrm{Te}_{20} \mathrm{Sn}_{2} \mathrm{Se}_{78-x} \mathrm{~Pb}_{x}(0 \leq x \leq 6)$ system. 


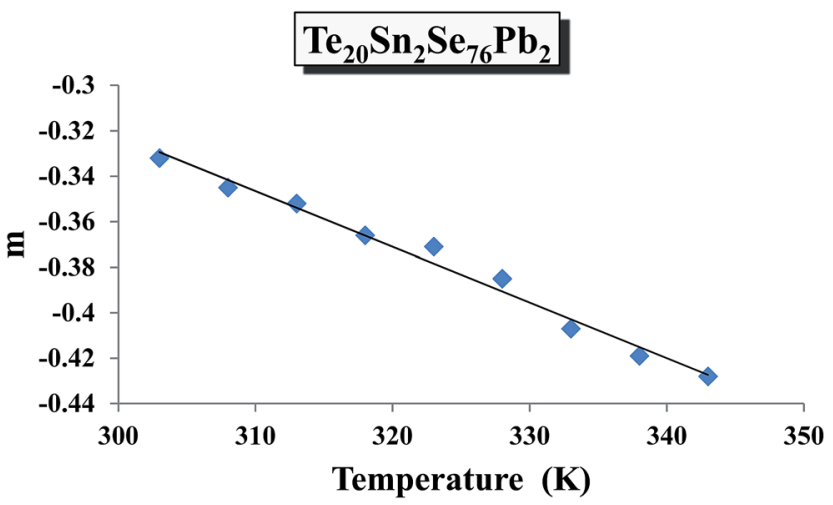

Fig. 10 Temperature dependence of frequency exponent $m$ of dielectric loss $\varepsilon^{\prime \prime}$ for the glassy $\mathrm{Te}_{20} \mathrm{Sn}_{2} \mathrm{Se}_{78} \mathrm{~Pb}_{2}$ alloy.

Thus, the morphological studies indicated that the acceptance of $\mathrm{Pb}$ into the host material was saturated at 6 at. $\mathrm{wt} \%$ of $\mathrm{Pb}$.

\subsection{Study of dielectric relaxation}

Dielectric analysis measures the electrical properties of materials as a function of temperature and frequency. There are two fundamental electrical characteristics of materials that are defined by the dielectric analysis. The first one is the capacitive insulating nature, which signifies its ability to store electrical charge. Second is the conductive nature, which represents the ability of motion of the electrical charges in the material. In the following section, we report the investigation on the dielectric properties of the newly designed multi-component glassy $\mathrm{Te}_{20} \mathrm{Sn}_{2} \mathrm{Se}_{78-x} \mathrm{~Pb}_{x}(0 \leq x \leq 6)$ system in the $50 \mathrm{kHz}$ to $1 \mathrm{MHz}$ frequency range and in the 303-348 $\mathrm{K}$ temperature range. The temperature and frequency dependence of dielectric constant and dielectric loss were investigated. Furthermore, we discussed the results of the variation in a.c. conductivity against applied field frequency and different glass compositions.

4.2.1 Frequency and temperature dependence of dielectric constant $\left(\varepsilon^{\prime}\right)$. Frequency dependence of the dielectric constant $\left(\varepsilon^{\prime}\right)$ for the present quaternary ChGS prepared by the melt quenching technique is shown in Fig. 6. The figure shows that the dielectric constant $\left(\varepsilon^{\prime}\right)$ decreases with increased applied frequency. The frequency decrease $\left(\varepsilon^{\prime}\right)$ can be attributed to the fact that at low frequencies for polar materials, the change in the dielectric constant is due to a contribution of the diverse constituents (electronic, ionic, dipolar or orientational and space charge) of polarization. The sum of all these types of polarizations shows the total polarization of the dielectric
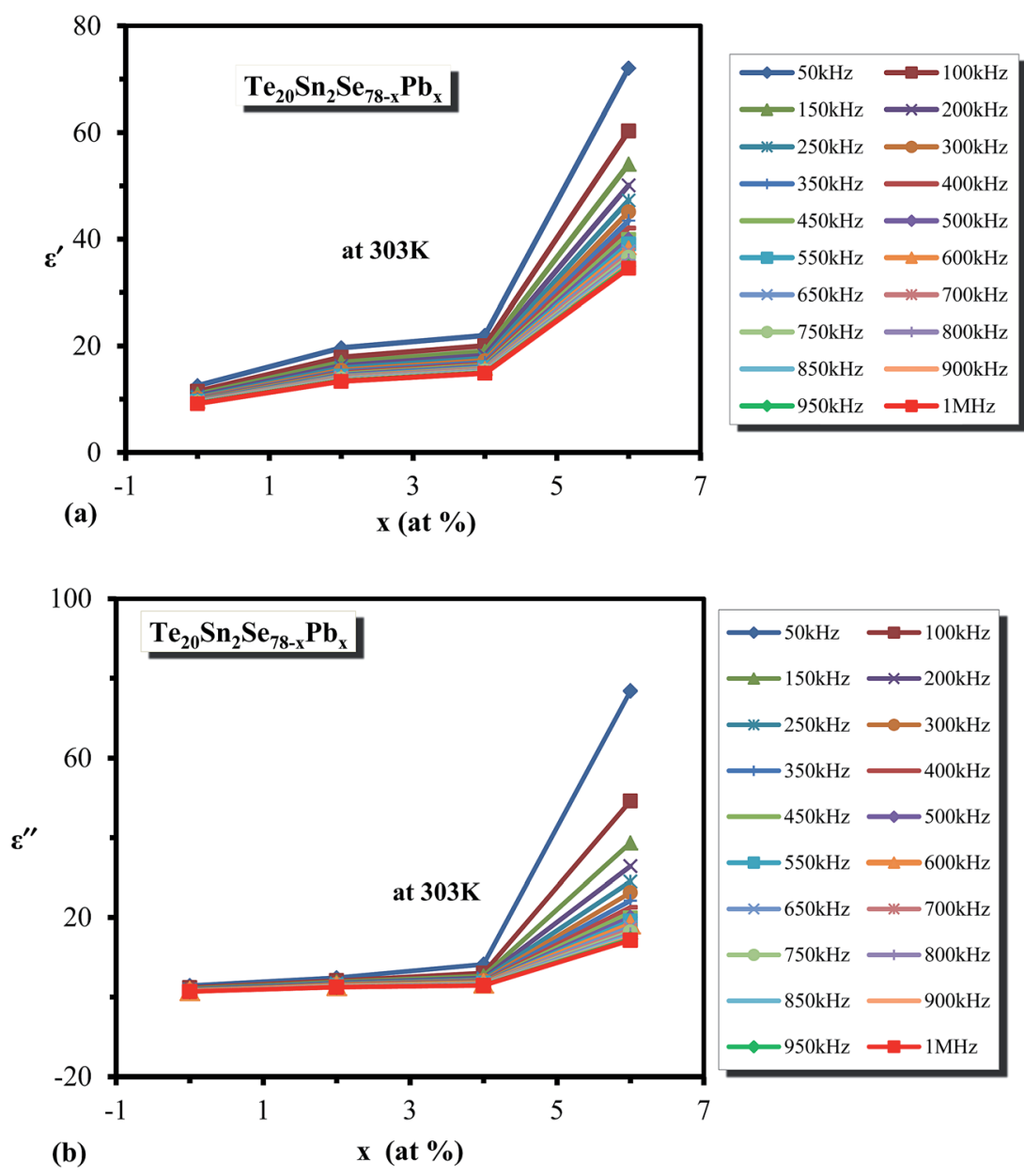

Fig. $11(\mathrm{a}, \mathrm{b})$ Composition dependence of $\varepsilon^{\prime}$ and $\varepsilon^{\prime \prime}$ for the glassy $\mathrm{Te}_{20} \mathrm{Sn}_{2} \mathrm{Se}_{78-x} \mathrm{~Pb}_{x}(0 \leq x \leq 6)$ system at a particular temperature. 


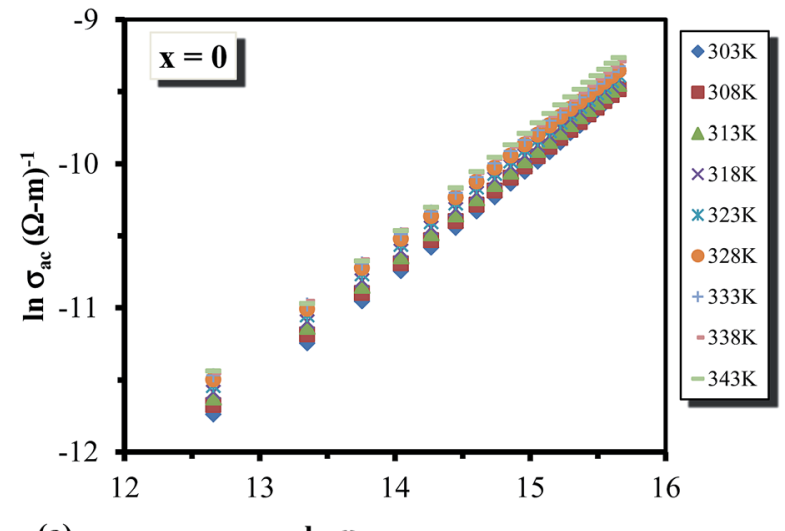

(a)

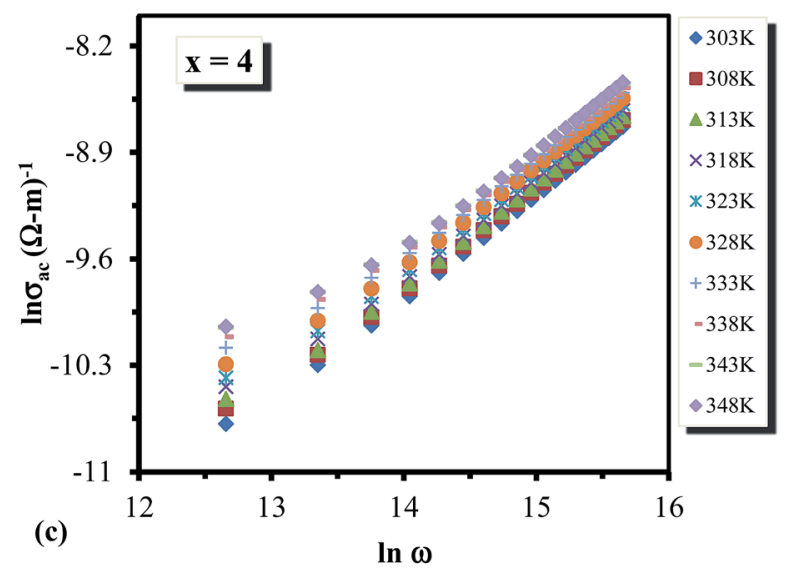

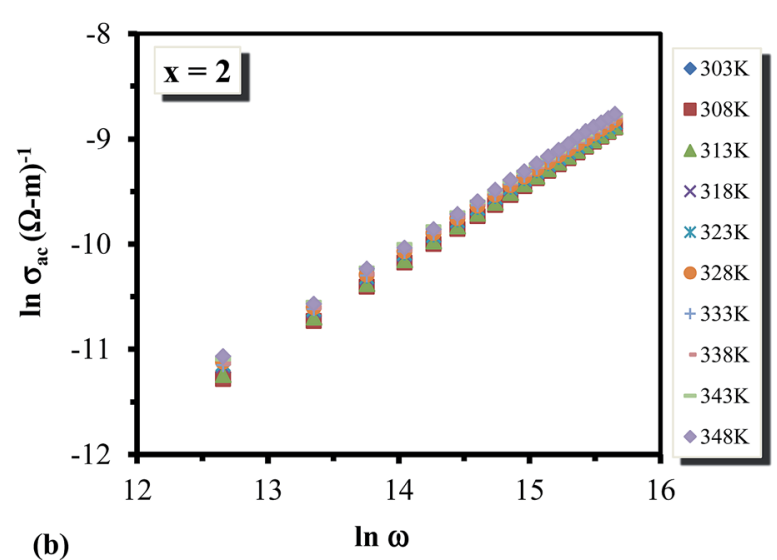

(b)

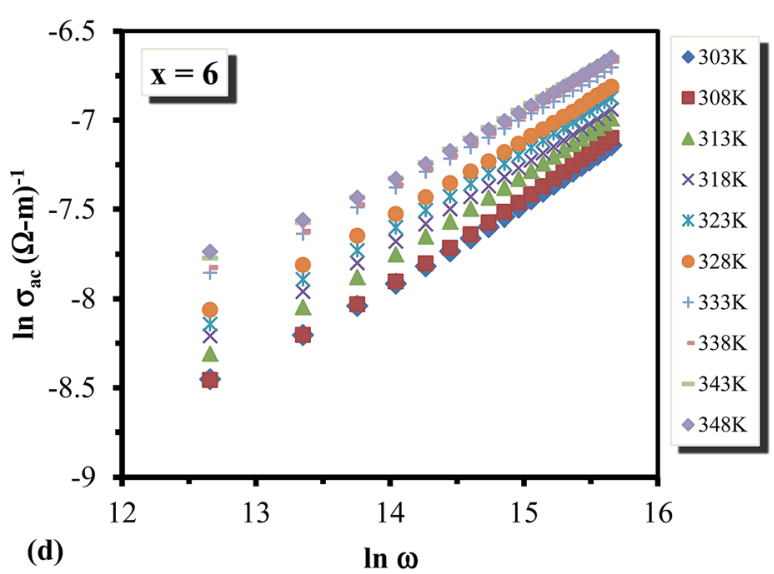

Fig. $12(a-d)$ Plots between $\ln \sigma_{\mathrm{ac}}(\omega)$ against $\ln \omega$ for the glassy $\mathrm{Te}_{20} \mathrm{Sn}_{2} \mathrm{Se}_{78-x} \mathrm{~Pb}_{x}(0 \leq x \leq 6)$ system.

materials. First is the electronic polarization, which occurs at frequencies up to $10^{16} \mathrm{~Hz}$. Next is the ionic type polarization, which exists on those materials in which negative and positive ions are present and happens due to displacement of these ions with respect to each other; this type of polarization happens at a frequency of $10^{13} \mathrm{~Hz}$. The third type of polarization is dipolar polarization, which takes place due to the presence of molecules that have permanent dipole moments and can change the direction of rotation to that of the applied electric field; this type of polarization happens at up to $10^{10} \mathrm{~Hz}$ frequencies. Finally, space charge polarization takes place due to the mobile charge of carriers by interfaces. Space charge polarization occurs at frequencies ranging between 1 and $10^{3} \mathrm{~Hz}$. Thus, the complete addition of all types of polarization represents the total polarization of the dielectric materials. In the present case, it is clear that the ionic type polarization does not show a pronounced effect on the total polarization, whereas the covalent nature of the studied compositions is expected. Therefore when the applied frequency is increased, the orientational polarization decreases because the dipoles no longer rotate sufficiently rapidly, and it takes more time compared to electronic and ionic polarizations; therefore their oscillations are behind those of the field. As the frequency is increased, the permanent dipole, if present in the medium, will be completely unable to follow the field and the orientational polarization will cease; consequently, the value of dielectric constant will decrease and approach a constant value at a higher frequency due to the interfacial or space charge polarization only.

Fig. 7 illustrates the temperature dependence of dielectric constant $\left(\varepsilon^{\prime}\right)$ at different frequencies in the abovementioned ranges for the glassy $\mathrm{Te}_{20} \mathrm{Sn}_{2} \mathrm{Se}_{78-x} \mathrm{~Pb}_{x}(0 \leq x \leq 6)$ system as a representative example. It is clear from this figure that dielectric constant $\left(\varepsilon^{\prime}\right)$ increases slowly at lower temperature, and after that, it increases appreciably with the temperature increase being different at different frequencies. This behavior was observed for all the glassy compositions under investigation. This increasing variation in dielectric constant $\left(\varepsilon^{\prime}\right)$ with temperature can be attributed to the fact that the dipoles in polar materials cannot orient themselves at low temperature. When the temperature is increased dipoles exist in the material, attain some freedom, i.e. the orientation of dipoles is facilitated, and hence increase the value of orientation polarization, which increases the value of dielectric constant $\left(\varepsilon^{\prime}\right)$. Thus the temperature dependence of $\varepsilon^{\prime}$ in the present study of a multicomponent chalcogenide glassy system is similar to that report by various scientists on other chalcogenide glasses..$^{35-37}$

4.2.2 Frequency and temperature dependence of dielectric loss $\left(\varepsilon^{\prime \prime}\right)$. Fig. 8(a-d)shows the dependence of dielectric loss $\left(\varepsilon^{\prime \prime}\right)$ with frequency at different temperatures from $303-348 \mathrm{~K}$ for the present multi-component chalcogenide glassy system. It is clear 


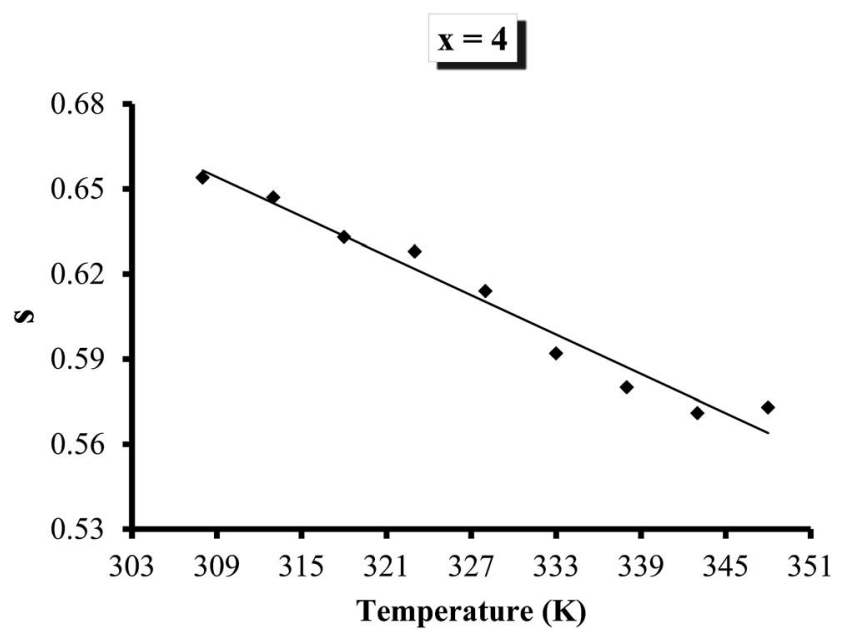

Fig. 13 Variation in frequency exponent $s$ of $\sigma_{\mathrm{ac}}$ with temperature for the glassy $\mathrm{Te}_{20} \mathrm{Sn}_{2} \mathrm{Se}_{74} \mathrm{~Pb}_{4}$ alloy.

from this figure that dielectric loss $\left(\varepsilon^{\prime \prime}\right)$ decreases with increasing applied frequency, and this behavior is different at different temperatures. The migration of ions in the material is the main cause of dielectric loss at low frequencies. Consequently, the dielectric loss shows high values at low and moderate frequencies, which is due to contributions from ion jump and conduction loss of ion migration, as well as the ion polarization loss. However, at high frequencies, the ion vibration may be the only cause of dielectric loss.

To explain the variation of dielectric loss against frequency, Mott et al. suggested an assumption ${ }^{\mathbf{3 8 , 3 9}}$ that when a sample is placed in an electric field, electron hopping takes place between the localized sites. The charge carriers moving between these sites hop from a donor to an acceptor state. In this respect, each pair of sites forms a dipole. Therefore, it can be revealed that the dielectric properties of chalcogenide materials can be deduced by regarding them as a set of dipoles, but only if the temperature is high enough; ${ }^{40}$ it was experimentally confirmed that under a definite temperature, the dielectric loss does not depend on the temperature. Moreover, it was supposed that each dipole has a relaxation time depending on its activation energy; ${ }^{\mathbf{1 1}, 42}$ which can be essentially attributed to the existence of potential barrier $W_{\mathrm{m}}$ over which the charge carriers must hop. ${ }^{43}$ The potential barrier, $W_{\mathrm{m}}$, which was proposed by Elliot, is due to the coulombic interaction between the neighboring sites forming a dipole. ${ }^{\mathbf{4 4 , 4 5}}$

Variation of dielectric loss $\left(\varepsilon^{\prime \prime}\right)$ with respect to temperature is shown in Fig. 9(a-d) at different frequencies for all the glassy alloys. It is clear from the figure that the dielectric loss $\left(\varepsilon^{\prime \prime}\right)$ increases with increasing temperature. According to Stevels, ${ }^{46}$ the dielectric relaxation phenomenon is divided into three parts, viz. dipole losses, conduction losses and vibrational losses. The conduction losses connect the migration of ions in excess of the large distance. The ions jump excess of the highest potentials in the glassy network. As the ions move, they give some of the energy to the lattice as heat, and the amount of heat lost per cycle is proportional to $\sigma_{\mathrm{ac}}(\omega) / \omega .^{\mathbf{4 6}}$
Therefore, the temperature increases above room temperature for the sample, consequently increasing the $\sigma_{\mathrm{ac}}(\omega) / \omega$, and thus the a.c. conduction loss increases amid increasing the temperature. At low temperatures, dielectric loss shows a minimum value, which may be due to the low values of conduction and dipole and vibration losses; however, at higher temperatures, all these three losses contribute to the dielectric loss. Hence it leads to an increase in dielectric loss with increasing temperature.

According to Guintini et al., ${ }^{31}$ the power $m$ in eqn (2) can be determined from the slopes of the straight lines by plotting $\ln \varepsilon^{\prime \prime}$ against $\ln \omega$ at different temperatures for all the compositions. Using such plots, the frequency exponent $m$ was determined for various samples at different temperatures. Temperature dependence of $m$ for a particular sample (glassy $\mathrm{Te}_{20} \mathrm{Sn}_{2} \mathrm{Se}_{76} \mathrm{~Pb}_{2}$ alloy) is shown in Fig. 10. This figure clearly reveals that the frequency exponent $m$ decreases with increasing temperatures. Similar results were obtained for other samples.

4.2.3 Composition dependence of dielectric constant $\left(\varepsilon^{\prime}\right)$ and dielectric loss $\left(\varepsilon^{\prime \prime}\right)$. In the case of the present multicomponent $\mathrm{Te}_{20} \mathrm{Sn}_{2} \mathrm{Se}_{78-x} \mathrm{~Pb}_{x}(0 \leq x \leq 6)$ glasses, the values of dielectric constant $\left(\varepsilon^{\prime}\right)$ and loss $\left(\varepsilon^{\prime \prime}\right)$ were increased with increasing the $\mathrm{Pb}$ concentration. The variation of $\varepsilon^{\prime}$ and $\varepsilon^{\prime \prime}$ with glass composition is shown in Fig. 11(a and b) for a particular temperature. Similar composition dependence was obtained at other temperatures in all the samples. Variation in these dielectric parameters with glass composition can be explained as follows:

When the concentration of $\mathrm{Pb}$ content increases, the glassy matrix becomes heavily cross-linked, and the steric hindrance increases. The Se-Se bond will be replaced by $\mathrm{Se}-\mathrm{Pb}$ bonds, which have higher bond energy as compared to the Se-Se bond since we incorporated $\mathrm{Pb}$ content in place of Se. Hence, the adhesive energy of the glassy matrix increases with increasing $\mathrm{Pb}$ content. Consequently, the increase in dielectric constant and dielectric loss takes place after the addition of $\mathrm{Pb}$ in parent ternary glass.

When iso-electronic atom Te is added to a-Se, the density of defect states is increased. ${ }^{47}$ This observation is explained on the basis of a structural defect model where Te is assumed to form positively charged impurities due to the low value of electro-negativity of Te compared to Se. ${ }^{48}$ When $\mathrm{Sn}$ atom, having lower electro-negativity in comparison of Se and Te atoms, is added to binary Se-Te system, ${ }^{48}$ it forms positively charged defect impurities. When $\mathrm{Pb}$ is introduced in the ternary

Table 1 Parameters used in curve fitting of the present topological glassy $\mathrm{Se}_{78-x} \mathrm{Te}_{20} \mathrm{Sn}_{2} \mathrm{~Pb}_{x}(x=0,2,4,6)$ semiconductors at room temperature for single-polaron and bi-polaron conduction

\begin{tabular}{lllrll}
\hline & & & \multicolumn{3}{c}{$N N_{\mathrm{p}}\left(\mathrm{m}^{-6}\right)$} \\
\cline { 5 - 6 }$x$ & $R_{\omega}(\AA)$ & $W_{\mathrm{m}}(\mathrm{eV})$ & \multicolumn{1}{c}{$\varepsilon$} & Single-polaron & Bi-polaron \\
\hline 0 & 18.6 & 0.96 & 9.7 & $8.2 \times 10^{53}$ & $7.2 \times 10^{56}$ \\
2 & 11.4 & 1.02 & 14.5 & $5.8 \times 10^{53}$ & $1.2 \times 10^{51}$ \\
4 & 15.4 & 0.79 & 16.1 & $6.3 \times 10^{53}$ & $3.8 \times 10^{51}$ \\
6 & 10.2 & 0.61 & 39.9 & $1.8 \times 10^{55}$ & $5.4 \times 10^{53}$
\end{tabular}




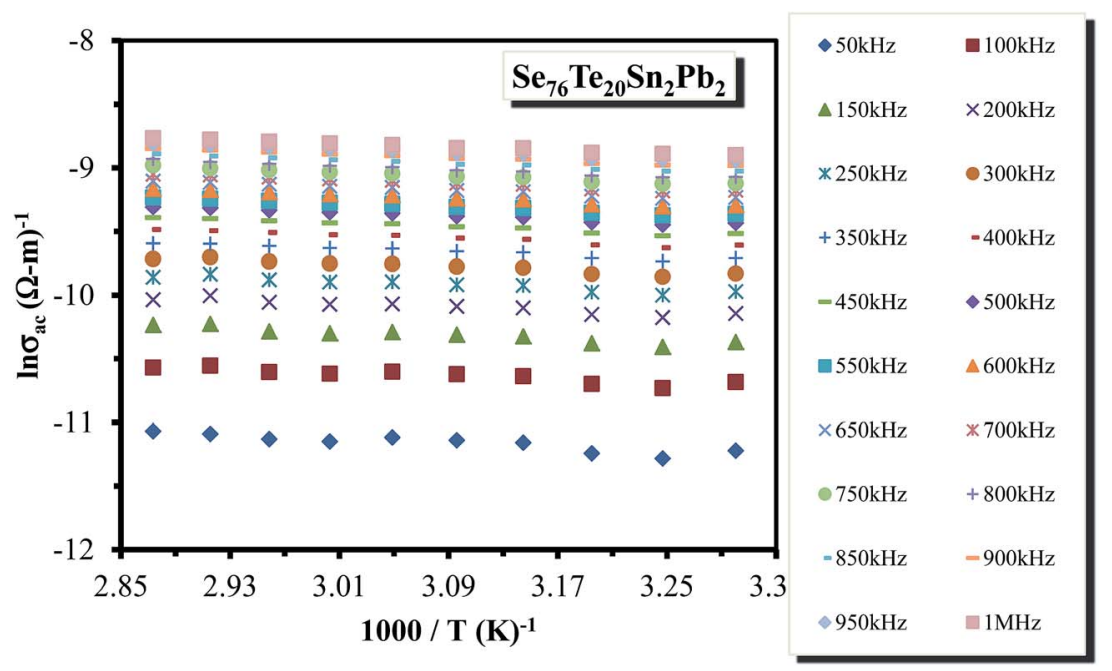

Fig. 14 Plots between In $\sigma_{\text {ac }}(\omega)$ against $1000 / T$ for the glassy $\mathrm{Te}_{20} \mathrm{Sn}_{2} \mathrm{Se}_{78} \mathrm{~Pb}_{2}$ alloy.

Se-Te-Sn system at the cost of Se, one can expect that the further positive charged defects states are generated because $\mathrm{Pb}$ possesses lower electro-negativity compared to Se. ${ }^{48}$ This causes an increase in the density of defect states in the glassy network of quaternary alloys compared to the parent ternary alloy. From the above discussion, we can conclude that adding $\mathrm{Pb}$ content into the parent $\mathrm{Se}_{78} \mathrm{Te}_{20} \mathrm{Sn}_{2}$ glass increases the number of charged defect states, which enhances the dielectric properties of materials.

\subsection{Analysis of thermally activated a.c. conduction}

4.3.1 Frequency dependence of a.c. conductivity. A common feature to all the amorphous materials is that the a.c. conductivity, $\sigma_{\mathrm{ac}}(\omega)$ varies with applied field frequency according to eqn (4). In this equation, the angular frequency exponent $s$ represents a temperature dependent constant, and generally its value is less than or equal to unity for most of the ChGS cases. The variation of a.c. conductivity with frequency at different temperatures for the aforementioned multicomponent glassy system is shown in Fig. 12(a-d). It is clear from this figure that the a.c. conductivity increased with increasing frequency at different temperatures. Another thing from this figure is that plots of $\ln \sigma_{\mathrm{ac}}(\omega)$ and $\ln \omega$ for all the compositions at different temperatures nearly coincided with each other. The weak temperature dependent mechanism in the present case may be ascribed to bi-polaron hopping between $\mathrm{D}^{+}$and $\mathrm{D}^{-}$centres around the Fermi level. ${ }^{44,45}$ Temperature dependence of $s$ for a particular sample (glassy $\mathrm{Te}_{20} \mathrm{Sn}_{2} \mathrm{Se}_{74} \mathrm{~Pb}_{4}$ alloy) is shown in Fig. 13. From this figure, it is clear that the frequency exponent $s$ is reduced with increasing temperature. Similar results were obtained for other samples. The temperature dependence of the frequency exponent $s$ indicates that a feasible conduction mechanism in the present ChGS is governed by correlated barrier hopping.

4.3.2 Temperature dependence of a.c. conductivity. The variation of a.c. conductivity with temperature in the present

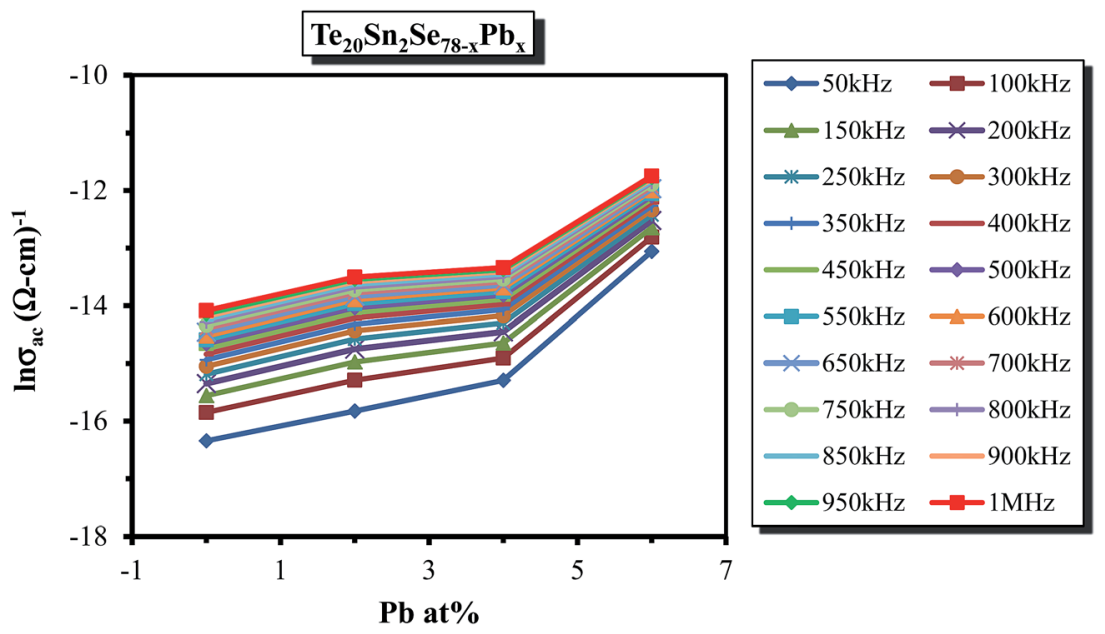

Fig. 15 Composition dependence of $\sigma_{\mathrm{ac}}$ for the glassy $\mathrm{Te}_{20} \mathrm{Sn}_{2} \mathrm{Se}_{78-x} \mathrm{~Pb}_{x}(0 \leq x \leq 6)$ system at a particular temperature (303 $\mathrm{K}$ ). 

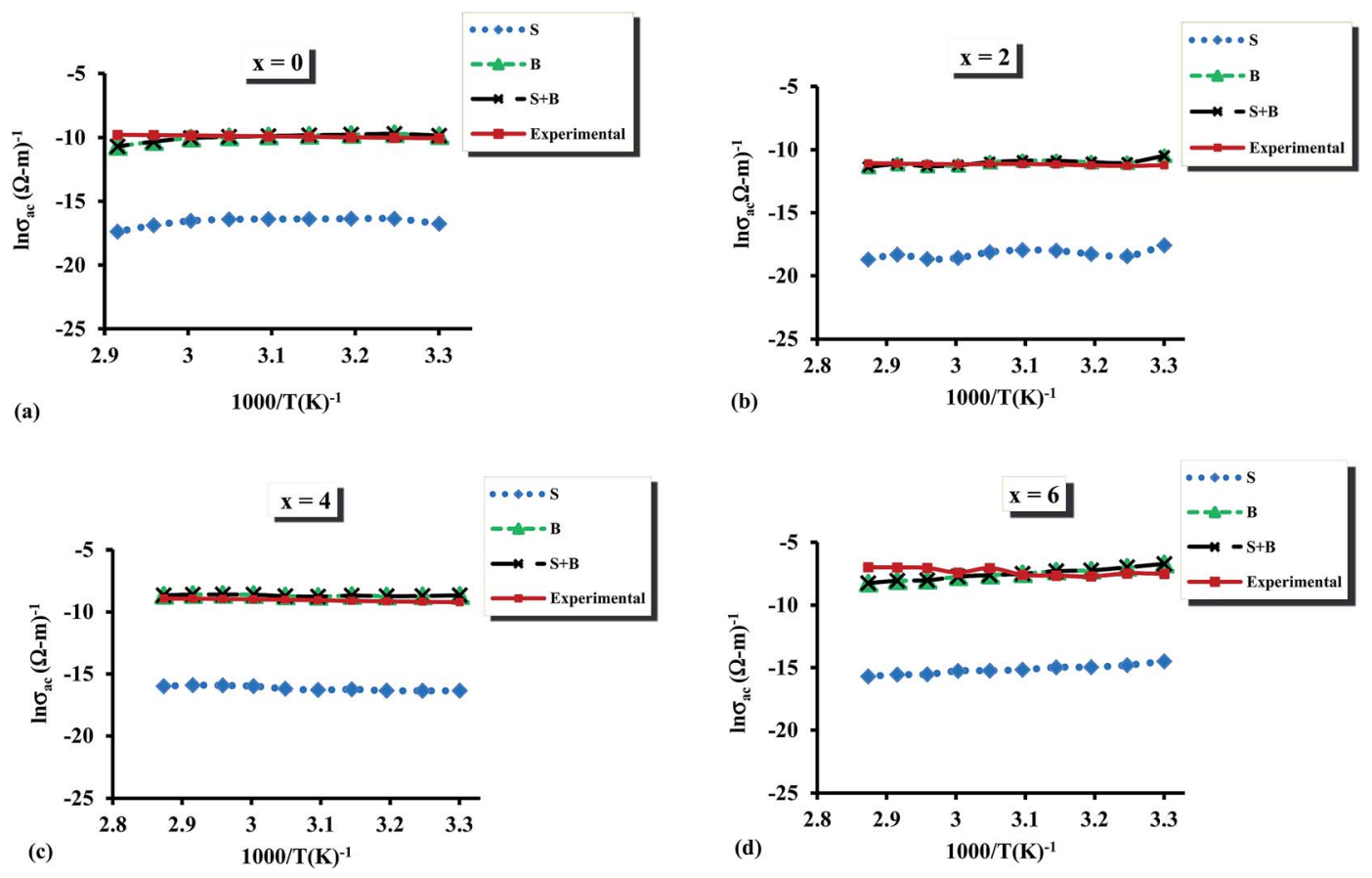

Fig. $16(a-d)$ Comparative analysis of theoretical and experimental a.c. conductivity for the glassy $\operatorname{Te}_{20} \mathrm{Sn}_{2} \mathrm{Se}_{78-x} \mathrm{~Pb}_{x}(0 \leq x \leq 6) \mathrm{system}$ at 500 $\mathrm{kHz}$.

samples of ChGS was investigated at diverse audio frequencies. The a.c. conductivity showed noticeable variation with temperature, and the plots of $\ln \sigma_{\mathrm{ac}}$ against $1000 / T$ were almost straight lines at different frequencies. Such plots are shown in Fig. 14 for a particular sample (glassy $\mathrm{Te}_{20} \mathrm{Sn}_{2} \mathrm{Se}_{76} \mathrm{~Pb}_{2}$ alloy). Similar results were obtained for other samples. The abovementioned results show that the a.c. conductivity varies exponentially with temperature according to the following equation:

$$
\sigma_{\mathrm{ac}}=\sigma_{0} \exp \left(-\frac{E_{\mathrm{ac}}}{k T}\right)
$$

It is evident from above relation that a.c. conduction in present samples of ChGS is a thermally activated phenomenon that follows Arrhenian temperature dependence.

4.3.3 Composition dependence of a.c. conductivity. Moreover, the a.c. conductivity in the glassy network of the parent glass $\mathrm{Se}_{78} \mathrm{Te}_{20} \mathrm{Sn}_{2}$ is increased with increasing the $\mathrm{Pb}$ concentration (see Fig. 15). This can be explained as follows:

In Se based glasses, there is tendency to form polymerized network glasses, and the homo-polar bond is qualitatively concealed. Therefore, when $\mathrm{Pb}$ was added into the host glassy material, the glassy matrix became heavily crosslinked and steric hindrance increased. Furthermore, replacing Se-Se bonds by Se-Pb bonds (which have higher bond energy than $\mathrm{Se}^{-} \mathrm{Se}^{48}$ ) caused an increase in the adhesive energy after the inclusion of $\mathrm{Pb}$ content. This is probably the reason for an increase in the a.c. conductivity after the inclusion of $\mathrm{Pb}$ content into the host material.

4.3.4 Estimation of density of charged defect states. According to the CBH model, single-polaron and bi-polaron hopping contributes to total a.c. conduction in ChGS. Keeping this experimental fact in mind, fitting of the experimental data was done in accordance with the CBH model mentioned in Section 3. For this, we adjusted the product $N N_{\mathrm{p}}$ of $N$ and $N_{\mathrm{p}}$ to achieve good agreement between the theoretical and experimental curves of $\ln \sigma_{\text {ac }}(\omega)$ versus $1 / T$. The fitting is at a particular frequency and identical fitting parameter values were used for other frequencies (see Table 1).

Fig. 16 shows the results of fitting of theoretical and experimental results in terms of the contributions for single-polaron and bi-polaron hopping and their sum. From Fig. 16, one can easily see that the theoretical curves are in good agreement with the experimental results. It is also evident that the contribution of single polaron hopping is much smaller than that of bipolaron hopping. The estimated values of density of charged

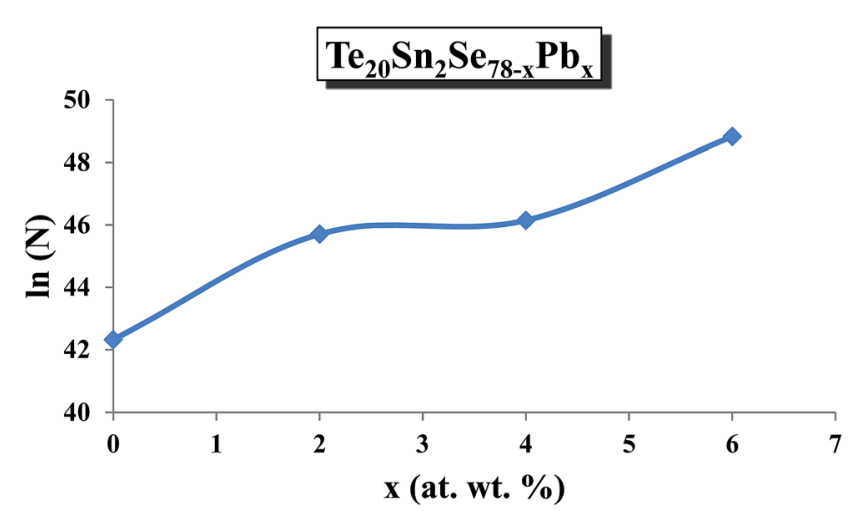

Fig. 17 Composition dependence of density of defect states $\sigma_{\mathrm{ac}}$ for the glassy $\mathrm{Te}_{20} \mathrm{Sn}_{2} \mathrm{Se}_{78-x} \mathrm{~Pb}_{x}(0 \leq x \leq 6)$ system. 
defect states are determined using these plots and the composition dependence of $N$ is shown in Fig. 17.

It is evident from this figure that the density of charged defect states increased with increasing $\mathrm{Pb}$ content in the host material. In the previous section, we observed that the dielectric loss also increased after adding $\mathrm{Pb}$ content in the host material.

It was reported ${ }^{37}$ that the dielectric loss in these materials mainly depends on the total number of localized sites. Thus, the increase of dielectric loss with the increased $\mathrm{Pb}$ content can be comprehended in terms of the increased density of defect states after inclusion of $\mathrm{Pb}$ content to the parent composition. The dielectric constant is also likely to rise with $\mathrm{Pb}$ content, as was observed in the present study, and this may be due to the increase in the number of dipoles at the higher $\mathrm{Pb}$ concentration. This is probably the reason for the increase in the value of density of defect states with increasing $\mathrm{Pb}$ content.

\section{Conclusions}

Temperature and frequency dependence of dielectric constant and dielectric loss were studied for the glassy $\mathrm{Se}_{78-x} \mathrm{Te}_{20} \mathrm{Sn}_{2} \mathrm{~Pb}_{x}$ $(x=0,2,4,6)$ system. The obtained experimental results were carried out through the 303-348 $\mathrm{K}$ temperature range and 50 $\mathrm{kHz}$ to $1 \mathrm{MHz}$ frequency range. It was found that the dielectric constant and dielectric loss both decreased with increasing frequency and increased with increasing temperature. It is also concluded that frequency and temperature dependence of the dielectric constant were attributed to the interfacial and orientational polarization, respectively. The temperature and frequency dependence of dielectric loss was linked to the conduction loss. Dielectric constant and loss both increased with increasing $\mathrm{Pb}$ content in the Se-Te-Sn composition. It was also found that a.c. conductivity did not show a strong temperature dependence. In addition, a.c. conductivity increased with increasing the $\mathrm{Pb}$ content into the parent composition. This happens due to the cross linking after inclusion of $\mathrm{Pb}$ content into the parent alloy and increases the adhesive energy of quaternary composition. Furthermore, the density of defect states for this system was also determined by fitting electrical a.c. conductivity data. Density of defect states increased after incorporating $\mathrm{Pb}$ in the parent $\mathrm{Se}_{78} \mathrm{Te}_{20} \mathrm{Sn}_{2}$ alloy.

\section{Acknowledgements}

NM is thankful to the Department of Science and Technology (DST), New Delhi, India for providing financial assistance under the Fast Track Young Scientists Scheme [Scheme No. SR/FTP/ PS-054/2010].

\section{References}

1 K. Michel, B. Bureau, C. Boussard-Pledel, T. Jouan, J. L. Adam, K. Staubmann and T. Baumannc, Sens. Actuators, B, 2004, 101, 252-259.

2 N. Mehta, J. Sci. Ind. Res., 2006, 65, 777-786.

3 N. Mehta and A. Kumar, Recent Pat. Mater. Sci., 2013, 6, 5967.
4 N. Mehta, Reviews in Advanced Sciences and Engineering, 2015, 4, 173-182.

5 H. F. Hamann, M. O'Boyle, Y. C. Martin, M. Rooks and H. K. Wickramasinghe, Nat. Mater., 2006, 5, 383-387.

6 M. Wuttig and N. Yamada, Nat. Mater., 2007, 6, 824-833.

7 D. Lencer, M. Salinga, B. Grabowski, T. Hickel, J. Neugebauer and M. Wuttig, Nat. Mater., 2008, 7, 972-977.

8 J. A. Moon, L. E. Busse, J. S. Sanghera and I. D. Aggarwal, Chalcohalide based infrared optical fibers, US6195483, 2001.

9 Deepika and N. S. Saxena, J. Phys. Chem. B, 2010, 114, 28-35.

10 H. Kumar and N. Mehta, Glass Phys. Chem., 2013, 39, 490498.

11 B. Vaidhyanathan, S. Murugavel, S. Asokan and K. J. Rao, J. Phys. Chem. B, 1997, 101, 9717-9726.

12 P. Dziawa, B. J. Kowalski, K. Dybko, R. Buczko, A. Szczerbakow, M. Szot, E. Lusakowska, T. Balasubramanian, B. M. Wojek, M. H. Berntsen, O. Tjernberg and T. Story, Nat. Mater., 2012, 11, 1023-1027. 13 Y. Okada, M. Serbyn, H. Lin, D. Walkup, W. Zhou, C. Dhital, M. Neupane, S. Xu, Y. Jui Wang, R. Sankar, F. Chou, A. Bansil, M. Z. Hasan, S. D. Wilson, L. Fu and V. Madhavan, Science, 2013, 341, 1496-1499.

14 I. Zeljkovic, Y. Okada, M. Serbyn, R. Sankar, D. Walkup, W. Zhou, J. Liu, G. Chang, Y. J. Wang, M. Z. Hasan, F. Chou, H. Lin, A. Bansil, L. Fu and V. Madhavan, Nat. Mater., 2015, 14, 318-324.

15 S. Devautour, C. P. E. Varsamis, F. Henn, E. I. Kamitsos, J. C. Giuntini and J. Vanderschueren, J. Phys. Chem. B, 2001, 105, 5657-5662.

16 B. Barrau, P. Demont, A. Peigney, C. Laurent and C. Lacabanne, Macromolecules, 2003, 36, 5187-5194.

17 L. Carpentier, M. Paluch and S. Pawlus, J. Phys. Chem. B, 2011, 115, 1062-1066.

18 M. Frumar and T. Wagnar, Curr. Opin. Solid State Mater. Sci., 2003, 7, 117-126.

19 A. Sharma, N. Mehta and A. Kumar, J. Mater. Sci., 2011, 46, 4509-4516.

20 M. Mokni, A. Kahouli, F. Jomni, J. L. Garden, E. Andre and A. Sylvestre, J. Phys. Chem. A, 2015, 119, 9210-9217.

21 L. E. Johnson, S. J. Benight, R. Barnes and B. H. Robinson, J. Phys. Chem. B, 2015, 119, 5240-5250.

22 R. D. Schaller and V. I. Klimov, Phys. Rev. Lett., 2004, 92, 186601.

23 R. J. Ellinson, M. C. Beard, J. C. Johnson, P. Yu, O. I. Micic, A. J. Nozik, A. Shabaev and A. L. Efros, Nano Lett., 2005, 5, 865-871.

24 S. A. McDonald, G. Konstantatos, S. Zhang, P. W. Cyr, E. J. D. Klem, L. Levina and E. H. Sargent, Nat. Mater., 2005, 4, 138-142.

25 M. T. Harrison, S. V. Kershaw, M. G. Burt, A. L. Rogach, A. Kornowski, A. Eychmuller and H. Weller, Pure Appl. Chem., 2000, 72, 295-307.

26 D. V. Talapin and C. B. Murray, Science, 2005, 310, 86-89.

27 P. Kania and S. Civis, Spectrochim. Acta, Part A, 2003, 59, 3063-3074.

28 M. Lach-hab, D. A. Papaconstantopoulos and M. J. Mehl, J. Phys. Chem. Solids, 2002, 63, 833-841. 
29 D. R. Goyal, S. Walker and K. K. Srivastava, Phys. Status Solidi A, 1981, 64, 35-357.

30 A. C. Wright, J. Non-Cryst. Solids, 2014, 401, 4-26.

31 J. C. Guintini, J. V. Zanchetta, D. Jullien, R. Eholie and P. Houenou, J. Non-Cryst. Solids, 1981, 45, 57-62.

32 S. R. Elliot, Philos. Mag., 1977, 36, 1291-1304.

33 K. Shimakawa, Philos. Mag. B, 1982, 46, 123-135.

34 S. R. Elliot, Adv. Phys., 1987, 36, 135-217.

35 M. W. Barsoum, Fundamentals of Ceramics, McGraw-Hill, New York, 1997, p. 585.

36 V. Modgil and V. S. Rangra, Phys. B, 2014, 445, 14-23.

37 J. Sharma and S. Kumar, Pramana, 2010, 74, 411-420.

38 N. F. Mott, E. A. Davis and R. A. Street, Philos. Mag., 1975, 32, 961-996.

39 R. A. Street and N. F. Mott, Phys. Rev. Lett., 1975, 35, 12931296.
40 K. K. Srivastava, A. Kumar, O. S. Panwar and L. N. D. Lakshminarayan, J. Non-Cryst. Solids, 1979, 33, 205-224.

41 A. E. Stearn and H. Eyring, J. Chem. Phys., 1937, 5, 113-123. 42 S. Glasstone, K. J. Laidler and H. Eyring, The theory of rate processes, McGraw Hill, New York, 1941.

43 P. Pollak and G. E. Pike, Phys. Rev. Lett., 1972, 28-22, 14491451.

44 S. R. Elliott, Philos. Mag. B, 1978, 37, 553-560.

45 S. R. Elliott, Solid State Commun., 1978, 27, 749-751.

46 J. M. Stevels, The Electrical Property of Glasses, in Hnadbuch der Physik, ed. S. W. Flugge, Springer, Berlin, 1997, vol. 20, p. 350.

47 A. Onozuka, O. Oda and I. Tsuboya, Thin Solid Films, 1987, 149, 9-15.

48 L. Pauling, J. Am. Chem. Soc., 1932, 54, 3570-3582. 